\title{
„Besser zeigen ihn seine Bücher“ Gelehrte im Porträt - eine Basler Tradition
}

\author{
VON ANDREAS BEYER
}

Nikolaus Meier gewidmet

Das Gelehrtenbildnis zählt zu den Bildgattungen, deren Entstehung lange zurückreicht und deren Konjunktur ungebrochen anhält. Kein Bericht über eine wissenschaftliche Sensation, die ohne eine Fotografie des verantwortlichen Forschers auskäme; kein Standardwerk großer Denker aus der Geschichte, dessen Umschlag nicht ein frühes Porträt schmückte. Das Bedürfnis nach Gelehrtenbildern ist gleichsam unversiegbar und so alt wie die Gelehrsamkeit selbst. Die Antike hat eine einschlägige, eigene Bildtradition ausgebildet, die über das Mittelalter bis in die Neuzeit reicht ${ }^{1}$; zur wirklichen Inkunabel dieses spezifischen Genres ist Antonello da Messinas Darstellung des „Hl. Hieronymus im Gehäuse" aus der Mitte der siebziger Jahre des 15. Jahrhunderts geraten, die heute in der Londoner National Gallery aufbewahrt wird.

Dabei hat die Frage nach der Bildwürdigkeit oder auch nach der Bildfähigkeit des Gelehrten, hat die Debatte um den Vorrang von

1 Der Mensch der römischen Antike, hg. v. A. Giardina, Frankfurt am Main 1991; A. von Hülsen-Esch, Gelehrte im Bild. Repräsentation, Darstellung und Wahrnehmung einer sozialen Gruppe im Mittelalter, Göttingen 2006; R. Burgess, Portraits of Doctors and Scientists in the Wellcome Institute of the History of Medicine, London 1973; K. Löcher, Humanistenbildnisse - Reformatorenbildnisse. Unterschiede und Gemeinsamkeiten, in: Literatur, Musik und Kunst im Übergang vom Mittelalter zur Neuzeit, hg. v. Boockmann, Göttingen 1995, 352-390; V. Valter, Gelehrte Gesellschaft. Wissenschaftler und Erfinder im Porträt, in: Erkenntnis, Erfindung, Konstruktion. Studien zur Bildgeschichte von Naturwissenschaft und Technik vom 16. bis zum 19. Jahrhundert, hg. v. H. Holländer, Berlin 2000, 833-859; R. Kanz, Dichter und Denker im Porträt. Spurengänge zur deutschen Porträtkultur des 18. Jahrhunderts, München 1993. - Abb. 1, 2, 3, 5 aus dem Archiv des Autors. 
Schrift oder Bild, die Gelehrtenwelt selbst vital beschäftigt. Prominent dafür steht der Name des Dichters und humanistischen Denkers Giovanni Boccaccio, der im 14. Jahrhundert entschieden gegen die zunehmende Bildersucht seiner Zeitgenossen zu Felde zog. Er hat sich nicht gewünscht, dass sein Dichterfreund Francesco Petrarca in einem Bildnis überdauere, denn er war davon überzeugt, dass dieser nach seinem Tod in seinen Schriften weiterlebe und so, überzeitlich, bei jeder Lektüre aufs Neue ins Leben der Nachwelt trete ${ }^{2}$. Als Schriftsteller hat Boccaccio vielleicht naturgemäß der Erinnerungskraft des Wortes den Vorrang vor jener des Bildes eingeräumt; der leibliche Körper des Dichters mochte ihm sterblich erscheinen, nicht aber das Corpus seines Werks. Und doch hat auch im Falle Petrarcas, von dem schon zeitgenössische Porträts existierten, das Bildbedürfnis gesiegt - noch unlängst. Ein einschlägig ausgewiesener Paläontologe hat sich im Frühjahr 2004, dem Jahr, in dem sich Petrarcas Geburt zum siebenhundertsten Male jährte, daran gemacht, dessen Grab in Arquà Petrarca zu öffnen, um aus den knöchernen Überresten und mit Hilfe avancierter, bildgebender Verfahren, Petrarcas wirkliches Antlitz zu rekonstruieren. Die Öffnung des Grabs hat freilich nur einen Schädel zutage gefördert, der nach Ansicht des Experten insgesamt zu klein ist und wohl eher einer Frau zugehörig gewesen sein muss. Eine Geschichte, die ganz nach Boccaccios Geschmack gewesen sein dürfte.

Der Ruhm, zumal jener, der sich an Bildnisse heftet, zählt zu den Prekaria, den widerruflichen, nicht einklagbaren Rechten. Und auch das Gelehrtenlob ist ein launiges Geschäft. Dass der Begriff der „fama“ zweideutig ist, nämlich Ruhm und Gerücht gleichermaßen bedeuten kann, ist dafür ein ebenso aussagekräftiges Zeugnis wie der Umstand, dass in der weit über den italienischen Sprachraum hinaus gängigen Formulierung „fortuna critica“ die Rezeptionsgeschichte eines Künstlers oder Gelehrten an die Glücks- und Schicksalsgöttin, Fortuna eben, gebunden bleibt. Der Künstlerbiograph Giovanni Battista Passeri hat das gegen Ende des 17. Jahrhunderts in die Worte gefasst: „Der Flug der Fama hängt vielfach auch vom Zufall ab, und vor allem bedarf es der

2 G. Boccaccio, De vita et moribus Francisci Petracchi de Florentia, a cura di R. Fabbri, in: Giovanni Boccaccio. Tutte le opere, a cura di V. Branca, Bd. 5.1., Milano 1992, 897-911. Zu den Petrarca-Porträts vgl. J. B. Trapp, Studies of Petrarch and his Influence, London 2003. 


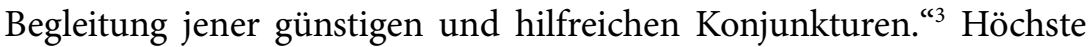
Wertschätzung zu Lebzeiten schließt postumes Vergessen bis hin zur „damnatio memoriae“ nicht aus. Dass die Schrift der verlässlichere Ruhmesverkünder ist, hat schon der Humanist Guarino da Verona im 15. Jahrhundert erkannt: „Wenn man schließlich die Hilfsmittel des Ruhmes vergleicht, so übertreffen die schriftlichen Annalen jedes beliebige Bild und jede Statue. Genau genommen zeigen die Bilder die Körper, die Annalen [die Schriften also] aber die Seele und die Sitten. Die Bilder sind stumm, aber die Annalen erfüllen mit ihrer Stimme Land und Meer. Die Bilder und Statuen können nur an wenigen Orten aufgestellt werden; die Annalen aber schweifen leicht über die ganze Welt hin und sind fähig, sich zu vervielfältigen. ${ }^{\text {" }}$

Und nicht nur eignet den Bildern schon materiell sozusagen eine geringere Erinnerungskraft; sie sind, wenn es sich um Porträts handelt, auch nicht immer gleichermaßen verlässlich. Schon deshalb begleitet das Porträt von früh an auch häufig das Wappen - etwa im Falle des von Albrecht Dürer verewigten Hieronymus Holzschuher (1526, Berlin, Gemäldegalerie) - von dem zuversichtlicher erwartet werden durfte, dass es wenigstens den genealogischen Stamm des Dargestellten zweifelsfrei identifizieren ließ.

Die recht unterschiedliche erinnerungsstiftende Qualität von Bildnis oder Wappen wird besonders deutlich am Porträt des Sebastian Brant (Abb. 3), das Hans Burgkmair um 1508 geschaffen hat. Das Porträt des Humanisten und Schriftstellers, Verfasser des „Narrenschiffs“ (Basel 1494) und von 1489 bis 1500 Professor beider Rechte an der Universität Basel, hat überhaupt erst nach der im Jahre 1949 erfolgten Entfernung der Übermalung des rückseitig angebrachten Wappenschildes identifiziert und mit Brant in Verbindung gebracht werden können.

Tatsächlich lässt die Heraldik Identifikationen leichter zu als die Physiognomie eines aus dem visuellen Gedächtnis der Zeit gefallenen Menschen. Es ließe sich auch schwerlich aus einem Vergleich etwa des Bildnisses von Burgkmair mit einer Porträtzeichnung Brants von der Hand Albrecht Dürers (Abb. 2) durch den Abgleich physiognomischer Korrespondenzen allein eine verbindliche Bestimmung des Dargestell-

3 G. B. Passeri, Die Künstlerbiographien, hg. v. J. Hess (Römische Forschungen der Bibliotheca Hertziana 11), Leipzig 1934, 72. Das Werk stammt von 1678.

4 Zitiert nach E. Garin, Geschichte und Dokumente der abendländischen Pädagogik, Hamburg 1966, Bd. 2, 205. 
ten vornehmen - denn auch die jeweilige Künstlerhandschrift tradiert ja nicht weniger den Maler oder Zeichner selbst.

Sebastian Brant posiert im Karlsruher Bildnis im scharfen Profil; ganz nach Art der antiken Herrscherdarstellungen auf Münzbildern, die hier besonders folgenreich auf die Bildpraxis eingewirkt haben. Er ist kostbar gewandet und trägt eine pelzverbrämte Kopfbedeckung; alles weist ihn als einem höheren Stand zugehörig aus. Und wirklich ist ja Brant hier schon in seiner Zeit als Stadtschreiber und Kanzler der freien Reichsstadt Straßburg porträtiert. Das Bildnis aber kommt weitgehend ohne Requisiten aus, ohne Symbole, schon gar solche der Macht. Vielmehr sind es die eingefallenen Wangen und der nach innen gekehrte Blick, die den Dargestellten als Denker und Schriftsteller auszeichnen, die Entbehrung und Askese sind die heraldischen Zeichen der Gelehrsamkeit.

Mit Burgkmair und Dürer, namentlich mit Sebastian Brant, berührt das Thema Basel, und wirklich darf das Gelehrtenbildnis eine Basler Tradition genannt werden, weil sich mit dem Bildnis Brants von der Hand Burgkmairs eine Gattung ausbildet, die vielfach an Basel gebunden bleiben wird - in unmittelbarer Folge ist es Erasmus von Rotterdam und sind es Hans Holbein d. J., Albrecht Dürer und Lucas Cranach d. Ä., die das Gelehrtenbildnis in immer anspruchsvollerem Gestus formulieren.

Anders als den anderen Bestellerkreisen von Porträts, denen es wie etwa im höfischen oder bürgerlichen Kontext - auch um ein augenfälliges dynastisches oder familiäres Fortleben ging, suchten die Humanisten zunächst und vor allem nach dem Ausdruck ihres eigenen, um es mit einem modernen Wort zu sagen: intellektuellen Selbstverständnisses, was nicht ausschließt, dass sich darin auch das Begehren nach Ruhm und Nachruhm und auch familiäre Durchsetzungsstrategien artikulieren. Eines der frühen Gelehrtenbildnisse, Lucas Cranachs um das Jahr 1503 entstandenes Porträt des Johannes Cuspinian (heute in der Sammlung Oskar Reinhardt „Am Römerholz“ in Winterthur), fungiert etwa als Pendantstück zu dem gleichzeitig geschaffenen Bildnis von dessen Frau Anna Putsch ${ }^{5}$. Und dennoch darf gelten, dass im Gelehrtenbildnis das grundsätzliche Dilemma bewahrt ist, von der Vergänglichkeit des eigenen Körpers zu wissen, sich im

5 Vgl. dazu A. Beyer, Das Porträt in der Malerei, München 2002, 118-122. 
geschriebenen Wort als Geist über den Körper zu behaupten, und doch zugleich anschauliches Zeugnis von der eigenen Existenz abzulegen.

Nicht selten dürften Gelehrten-Porträts wohl deshalb auch in graphischen Blättern überliefert sein, weil sie damit auf einer medialen Ebene, auf Papier und im Druck, mit dem materiellen Träger des schriftstellerischen Werks korrespondierten. Gleichwohl gilt, dass, wie etwa im berühmten Kupferstich Dürers aus dem Jahr 1526, der den Humanisten und Reformer Philipp Melanchthon abbildet, das Bewusstsein der Uneinholbarkeit des Geistes durch die Malerei vorherrschte. Dort begleitet folgende, fingiert in Stein gemeißelte Inschrift das im Profil wiedergegebene Bildnis: VIVENTIS POTUIT DURERIUS ORA PHILIPPI / MENTEM NON POTUIT PINGERE DOCTA MANUS („Das Antlitz des lebenden Philipp, nicht seine Geistseele vermochte Dürer mit gelehrter Hand zu malen") ${ }^{6}$. Es handelt sich um ein klassisches Bildepigramm, das von der Signatur des Malers gefolgt wird. Dass die Inschrift als steinernes Dokument erscheint, verweist auch hier auf die größere Dauer, die dem Wort vor dem Bild zukommt. Rudolf Preimesberger hat überzeugend auf die Ambivalenz, ja die Paradoxie verwiesen, die sich hier artikuliert. Der Künstler - der sich, indem er seine Hand eine „gelehrte" nennt, mit dem Gelehrten im Bild auf eine Stufe stellt - formuliert recht eigentlich den Triumph der Kunst, nämlich die Schwierigkeit, das eigentlich nicht Darstellbare (den Geist) in Gestalt des Körpers eben doch zur Ansicht, Inneres und Äußeres in eine anschauliche Wechselwirkung gebracht zu haben. Der Körper erst lässt überhaupt von dem ihm innewohnenden Geist sprechen ${ }^{7}$.

Es scheint in diesem Blatt all das auf, was die zeitgenössische und nachfolgende Bildnistradition insgesamt beschäftigen sollte - auch und vor allem in Basel. Dafür ist beredtes Beispiel das von Hans Holbein d. J. geschaffene Porträt des Bonifacius Amerbach aus dem Jahr 1519 (Abb. 4). Amerbach studierte seit 1512 Jura und klassische Sprachen in Freiburg (wo er eng mit Erasmus von Rotterdam verkehrte), zog dann nach Avignon weiter, wo er 1525 promoviert wurde. Das Porträt entstand vor seinem Weggang in die südfranzösische Universitätsstadt; der schneebedeckte Bergrücken im Bildhintergrund, eine

6 Vgl. Porträt, hg. v. R. Preimesberger u. a., Berlin 1999, 220 ff.

7 Ebd. 
Rarität in den ohnehin seltenen Landschaftsdarstellungen Holbeins, wird für die bevorstehende Reise über die Alpen stehen. Insofern steht das Bildnis in einer klassischen Tradition, die Porträts als erinnerungsstiftende Hinterlassenschaft der in die Ferne aufbrechenden Dargestellten kennt ${ }^{8}$. Als Bild aber kündet es vor allem von der erheblichen humanistischen Ambition des Dargestellten wie von seiner bildkritischen Expertise. Amerbachs Anteil an der Gestaltung des Porträts ist verbürgt; die Inschrift ist von ihm selbst verfasst worden, ein Entwurf dazu hat sich in der Basler Universitätsbibliothek erhalten. Sie besagt: PICTA LICET FACIES VI / VAE NON CEDO SED INSTAR / SVM DOMINI IVSTIS NO / BILE LINEOLIS: / OCTO IS DUM PERAGIT / TPIETH, SIC GNAVITER IN ME / ID QVOD NATVRAE EST, / EXPRIMIT ARTIS OPVS. // BON. AMORBACCHIVM. / IO. HOLBEIN. DEPINGEBAT. / A. M. D. XIX. PRID. EID. OCTOBR („Wenn auch nur ein gemaltes Gesicht, stehe ich doch dem lebendigen nicht nach, sondern bin ein Ebenbild meines Herrn, ausgezeichnet durch richtige Linien. Zum Zeitpunkt, da er acht Dreijahresabschnitte vollendet [24 Jahre alt wird], gibt das Kunstwerk mit Fleiß in mir das wieder, was sein Wesen ist. Jo[hannes] Holbein malte Bon[ifacius] Amerbach am 14. Oktober 1519") $)^{9}$. Der 14. Oktober war Amerbachs Geburtstag; er kommt in diesem Gemälde sozusagen noch einmal zur Welt.

Die quadratische Tafel zeigt einen nahezu frontalen Büstenausschnitt und gibt das Gesicht fast ganz im Profil wieder. Der Dargestellte ist kostbar und nüchtern zugleich gekleidet; der dunkle Ton seines Gewands und des Baretts rahmen das lebhafte Inkarnat seines Gesichts, das nichts von seiner leuchtenden Gegenwart eingebüßt hat. Das gesamte Bildnis lebt von Kontrasten. Bonifacius Amerbach posiert im Bildvordergrund. Ohne weiteres öffnet sich dahinter ein weiter Landschaftsausblick, von einem hohen Himmel überwölbt. Ein junger Mann, Leben und Welt vor sich, bürgerlich gerüstet. Doch vor die Erlangung dieser strahlenden Welt schieben sich, gleichsam anstelle des ausgelassenen Mittelgrunds, der seitlich flankierende Stamm eines Feigenbaums links und ein daraus in die rechte Bildhälfte hinein wachsender Ast, der den Dargestellten hinterfängt. Holbein, der später in London, namentlich für die Mitglieder des Stahlhofs, eine Vielzahl

8 Vgl. zur sog. Butades-Legende Beyer, Porträt (wie Anm. 5), 17.

9 Zitiert nach Hans Holbein d. J. - die Jahre in Basel 1515-1532, Katalog zur Ausstellung im Kunstmuseum Basel, 1.4.-2.7.2006, München 2006, 194. 
von Bildnissen energisch nach oben drängender junger Männer malen sollte, präludiert dieses Genre hier und folgt doch ganz den Regeln einer humanistisch inspirierten Balance zwischen Darstellungsbedürfnis und Demutsgestus.

Am Stamm des Baumes befindet sich die gerahmte, ebenfalls quadratische Holztafel samt oben zitierter Inschrift, die, einer Identitätskarte gleich, den Namen des Modells, sein Alter (24 Jahre) und seinen Geburtstag nennt. Zudem spricht die Inschrift davon, dass dieses Ebenbild zwar nur ein Gemälde, aber doch in „richtigen Linien“ geschaffen worden sei, und verschweigt auch nicht den Namen seines Malers. Sich täglich im Ziehen einer Linie zu üben, gehört zu den Topoi der antiken Künstlerbiographie. Die Inschrift lobt also Holbein, verweist aber in ihrer Relativierung und durch das Medium der Schrift selbst auf den Vorrang der Sprache, des Worts, vor der sinnlichen Malerei. Ohne sie wüssten wir kaum vom Namen des Dargestellten. Wie zur Bekräftigung dieser Entkräftigung ragt der Astrest, an dem die Tafel am Stamm befestigt ist, in Augenhöhe des Amerbach bedrohlich weit hervor: Dass dieser nicht vollends im Profil gegeben ist, sondern beide Augen sichtbar werden, erlaubt so, die Sehinstrumente wirkungsvoll daran zu erinnern, dem Trugbild nicht zu trauen und sich nicht vom Zeugnis der Schrift abzuwenden.

Auch der Feigenbaum artikuliert eine Mahnung. Er ist mit dem Sündenfall verbunden und Symbol von Vergänglichkeit. Daran, dass mitten im Leben der Tod wächst, erinnert der Ast den Dargestellten und mindert so den selbstgefälligen Gestus, der ihn ein Bildnis seiner selbst hat beauftragen lassen. In diesem Porträt Amerbachs - der als Universalerbe des Erasmus den Grundstock des nach seiner Familie benannten Kabinetts gelegt hat und mit dessen Namen das Fortdauern des Humanismus in Basel auch in den widrigen Zeiten des reformatorischen Umbruchs verbunden ist - artikuliert sich so schon früh dessen ebenso geschicktes wie wirkungsvolles Agieren. Er klagt in dieser Inkunabel der Bildpolitik sichtbare Präsenz, Wortführerschaft und Erinnerung an sich selbst ein und federt sie zugleich in Beschwörungen der Demut ab.

Der mit Amerbach so eng verkehrende Erasmus von Rotterdam hat sich der Bildnisproduktion nicht weniger strategisch bedient. Und wenn er auch bekanntlich ein durchaus entzündliches Verhältnis zur 
bildenden Kunst hatte ${ }^{10}$ und in einem Brief gefordert hat „Locere ut te videam" (rede, dass ich Dich sehen kann), womit dem Wort die größere Zeigekraft attestiert wird, so hat er sich doch in vielerlei Formaten abbilden lassen und sich wie kaum ein anderer auf den Einsatz des Porträts zu verstehen gewusst. Man darf uneingeschränkt behaupten, dass er die ikonische Propaganda zur Durchsetzung eines Lehrgebäudes auf gänzlich neue Grundlagen gestellt hat. Als Holbeins bedeutendstes Porträt des niederländischen Humanisten, der von 1514 bis 1529 und dann wieder von 1535 bis zu seinem Tode 1536 in Basel lebte und wirkte, darf jenes Bildnis aus dem Jahr 1523 gelten, das gemeinhin unter dem Titel „Erasmus mit einem Renaissancepilaster“ figuriert (Abb. 5). Der weitgehend unabhängig arbeitende Erasmus stand in weitverzweigtem Briefkontakt zu anderen Philosophen, vor allem aber auch zu Gönnern, die sein Auskommen sicherten. Diesen sandte er nicht selten auch sein Porträt zu, um dem wiederholt an ihn herangetragenen Wunsch nach seiner Gegenwart wenigstens durch ein stellvertretendes Bildnis nachzukommen. In diesem, aus Basel an William Warham, Erzbischof von Canterbury und großherziger Zuwendungsgeber des Erasmus, gesandten Gemälde, in dem sich die Individualität des Erasmus und die Ikonographie des Gelehrtenbildnisses monumental verbinden, wird zwar deutlich, dass Erasmus der Schrift durchaus eher zutraute, die eigene Sterblichkeit zu überdauern als dem Abbild. Aber ein dem Bild zugehöriger Brief bekundet doch zugleich seine Überzeugung, dass wenigstens ein Mindestmaß an Dauerhaftigkeit auch dem Porträt zugeschrieben werden darf: „Ich nehme an, dass Sie [...] eine gemalte Wiedergabe meiner Züge erhalten haben, so dass, sollte es Gott gefallen, mich von hier abzurufen, Sie doch ein Stück des Erasmus haben werden. " ${ }^{11}$

Erasmus steht als verlängerte Halbfigur in Dreiviertelansicht nach rechts gewandt. Er sitzt nicht, wie das die Bildtradition seit den frühen Darstellungen des Hl. Hieronymus gleichsam forderte, am Pult, sondern hat offenbar hinter einer steinernen Brüstung Aufstellung bezogen. Schon das unterstreicht den „Denkmalcharakter“ dieses Porträts. Der Philosoph ist in ungeschöntem Alter gezeigt, in zwei kostbare, pelzverbrämte Mäntel gekleidet und trägt ein dunkles Barett. Seine

10 Vgl. E. Panofksy, Erasmus and the Visual Arts, in: Journal of the Warburg and Courtauld Institutes 32 (1969), 200-227.

11 Zitiert nach Beyer, Porträt (wie Anm. 5), 122. 
Hände ruhen auf einem geschlossenen Buch, das auf der Brüstung lagert. Sein Titel korrespondiert mit keinem der Werke des Erasmus; vielmehr darf die auf dem Seitenschnitt verlaufende Inschrift HPAKL $\Lambda$ EIOI ПONOI ERAS[M]I ROTERO[DAMI] („Die herkulischen Arbeiten des Erasmus von Rotterdam") so gelesen werden, dass hier das Gesamtwerk des Erasmus mit den „Zwölf Mühen“ des Herkules, des für die Zeit verbindlichen antiken Tugendhelden, gleichgesetzt wird. So wie die Taten des Herkules erst dessen Vergöttlichung ermöglicht und ihm ewigen Ruhm eingetragen haben, so werden die Schriften des Erasmus diesem zu immerwährender Fama verhelfen. Die „Taten“ des Herkules waren sämtlich körperliche Kraftakte; die auffällig ruhenden Hände des Erasmus setzen so geistige und körperliche Leistung gleich. Es kann hier auf die zahlreichen Anspielungen, die in diesem Bildnis erkannt worden sind, nicht in der gebotenen Ausführlichkeit eingegangen werden ${ }^{12}$. Es handelt sich um ein in gelehrten Anspielungen und Verweisen überreiches Werk, das belegt, dass auch im Bild sich gedanklicher Anspruch kongenial artikulieren kann.

Erasmus blickt den Betrachter nicht an, er kommuniziert weder mit diesem, noch mit seinem eigenen Werk, so wie er das in den anderen Bildnissen, die ihn oft schreibend zeigen, tut. Sein Blick signalisiert vielmehr reflexive Versunkenheit, Innenblick und Rückschau, so wie ja auch die Anspielung auf die Taten des Herkules vermuten lassen darf, hier sei das irdische Tun als vollendet begriffen. Erasmus hat somit viele Jahre vor seinem Tod gleichsam ein ikonisches Testament malen lassen, das sein Adressat, Erzbischof Warham, seinerseits durch ein von Holbein anlässlich von dessen erster London-Reise, die wohl der Überbringung des Erasmus-Porträts diente, geschaffenes Bildnis beantwortete. Dieses Porträt (heute in der National Gallery in London) hat Erasmus nie erreicht. Es zeigt aber, wie hier zwischen zwei Theologen über das Bild kommuniziert wurde, wie wirkliches Bildhandeln operiert. Warham posiert dem Erasmus ganz vergleichbar, auch die Hände korrespondieren. Das Buch aber, auf dem sie ruhen, ist geöffnet. Es antwortet der Leser Warham dem Autor Erasmus.

Holbein hat bei seiner ersten Englandfahrt auch ein Bildnis des Thomas Morus angefertigt (heute in der Frick Collection, New York)

12 Vgl. dazu die Bibliographie in Beyer, Porträt (wie Anm. 5), 40o. Siehe auch S. Gronert, Bild - Individualität. Die Erasmus-Bildnisse von Hans Holbein d. J., Basel 1996. 
und zudem ein (heute verlorenes) Gruppenbildnis der Familie des Verfassers der "Utopia“ geschaffen. Die Vorzeichnung von Letzterem hat sich im Kupferstichkabinett des Basler Kunstmuseums erhalten; sehr wahrscheinlich entstammt sie dem Nachlass des Erasmus, der sich solcherart der visuellen Präsenz seines gelehrten Freundes und dessen Umfelds versicherte ${ }^{13}$. Auch das erlaubt, die Bedeutung Basels als Umschlagplatz eines transkontinentalen Gelehrtenbildnisverkehrs zu unterstreichen.

Es darf aber von Holbeins Erasmus-Bildnissen nicht gesprochen werden, ohne auch auf Dürers Erasmus-Porträt zu verweisen (Abb. 1). Dürer hat den Theologen gelegentlich getroffen (so in Basel 1520, wovon eine im Pariser Louvre aufbewahrte Zeichnung, ein DreiviertelPorträt des Erasmus, Zeugnis ablegt), und dieser hat früh schon den Ehrgeiz entwickelt, von dem damals unstrittig berühmtesten Maler des deutschen Sprachraums abgebildet zu werden. Dürers Stich aber ist erst 1527 entstanden. Auch Dürer zeigt Erasmus stehend, hier aber an einem Schreibpult. Er figuriert im Talar des Gelehrten, das bartlose Gesicht erscheint im Halbprofil, eine Haube bedeckt sein Haupt. Erasmus ist im Begriff, einen Bogen Papier zu beschreiben, seine Linke hält das geöffnete Tintenfass. Dass im Bildvordergrund, auf einer Ablage, ein geöffnetes Buch und vier mit Schliessen verschlossene Bücher liegen, ist als Hinweis darauf gelesen worden, dass der Betrachter über den Gehalt der Schriften des Erasmus zu dessen äußerer Gestalt vordringen solle ${ }^{14}$.

Davon spricht auch die hochrechteckige, gerahmte Inschrifttafel, die mit den Proportionen des Kupferstichs korrespondiert und die als der zweite Protagonist des Blattes anzusehen ist. Auf ihr steht (auf Lateinisch) geschrieben: „Das Bild des Erasmus von Rotterdam von Albrecht Dürer nach der lebenden Erscheinung gezeichnet.“ Auf Griechisch folgt dann: „Das bessere werden die Schriften zeigen.“ Auch dieses Blatt ist jüngst von Rudolf Preimesberger aufschlussreich erörtert worden, zumal die auf Quentin Metsys zurückgehende Inschrift. Preimesberger hat in der doppelten und mehrfachen Antithese den

13 Vgl. Hans Holbein d. J. (wie Anm. 9), 370 ff.

14 Albrecht Dürer. Das druckgraphische Werk, hg. v. R. Schoch u. a., Bd. 1, München 2001, $218 \mathrm{ff}$. 
Schrift- und Bildbestandteil des Gelehrtenporträts in deren wechselseitigem Bezug verhandelt erkannt ${ }^{15}$.

Erasmus hat sich rasch beklagt, dass dieses Porträt dem Kriterium der Ähnlichkeit nicht gehorche, und sich kaum darin wieder erkennen wollen. Was überrascht, ist es doch Erasmus selbst, der die Künstler selbst wiederholt hat bekräftigen lassen, dass seinen Schriften die verlässlichere Abbildfähigkeit zukomme. Oskar Bätschmann hat treffend beobachtet, dass Erasmus von Dürer beim Schreiben eines Briefes gezeigt wird ${ }^{16}$. Er schreibe, so Bätschmann, an seinem Bild, umgeben von den teils verschlossenen Büchern, die sein unsichtbares Bild bewahrten. Alles ist in Dürers Blatt ineinander verschränkt; Wort und Bild treten in einen Paragone, der zuletzt Dürers Porträtkunst triumphieren lässt. Wie schon in seinem Melanchthon-Bildnis ist es nämlich seine "gelehrte" Hand, die das Undarstellbare in den Bereich der Sichtbarkeit überführt, wie dort bietet er zwei Bilder in einem: „den lesend Schauenden und schauend Lesenden. ${ }^{\text {17 }}$

Es hat sich von hier aus, von den Bildnissen des Sebastian Brant und des Erasmus von Rotterdam, eine Bildtradition gestärkt und entwickelt, die nicht mehr abreißen sollte. Jeder Gelehrte von einiger Reputation hat fortan Anspruch darauf erhoben, im Bild festgehalten zu werden - wohl wissend, dass sich das Bild nur komplementär zum schriftlichen Werk verhalten kann und dass die eigene physiognomische Unverwechselbarkeit nicht immer damit korrespondieren muss. Es braucht hier die lange Linie, die bis in unsere Zeit, bis hinein in die Professorengalerie des Museums in der Basler Augustinergasse reicht, gar nicht gezogen werden. Es soll aber abschließend mit einer Anekdote von dem Unabgegoltenen gesprochen werden, das zwischen der Erscheinung des Gelehrten und dem gelehrten Werk unausgesetzt bestehen bleibt. Als sich Jacob Burckhardt zur Anfertigung der rasch berühmt gewordenen Porträtfotografie (Abb. 6) im Sonntagsstaat zum verabredeten Zeitpunkt beim Fotografen einfand, soll er zunächst wieder fortgeschickt worden sein: man habe heute keine Zeit, beschied

15 Porträt (wie Anm. 6), 228-238. Vgl. auch P.-K. Schuster, Überleben im Bild. Bemerkungen zum humanistischen Bildnis der Lutherzeit, in Köpfe der Lutherzeit, hg. v. W. Hofmann, München 1983, 18-25.

16 O. Bätschmann/P. Griener, Holbein-Apelles. Wettbewerb und Definition des Künstlers, in: Zeitschrift für Kunstgeschichte 57 (1994), $643 \mathrm{f}$.

17 Porträt (wie Anm. 6), 237. 
man ihn, man erwarte einen bedeutenden Historiker zur Porträtsitzung. 


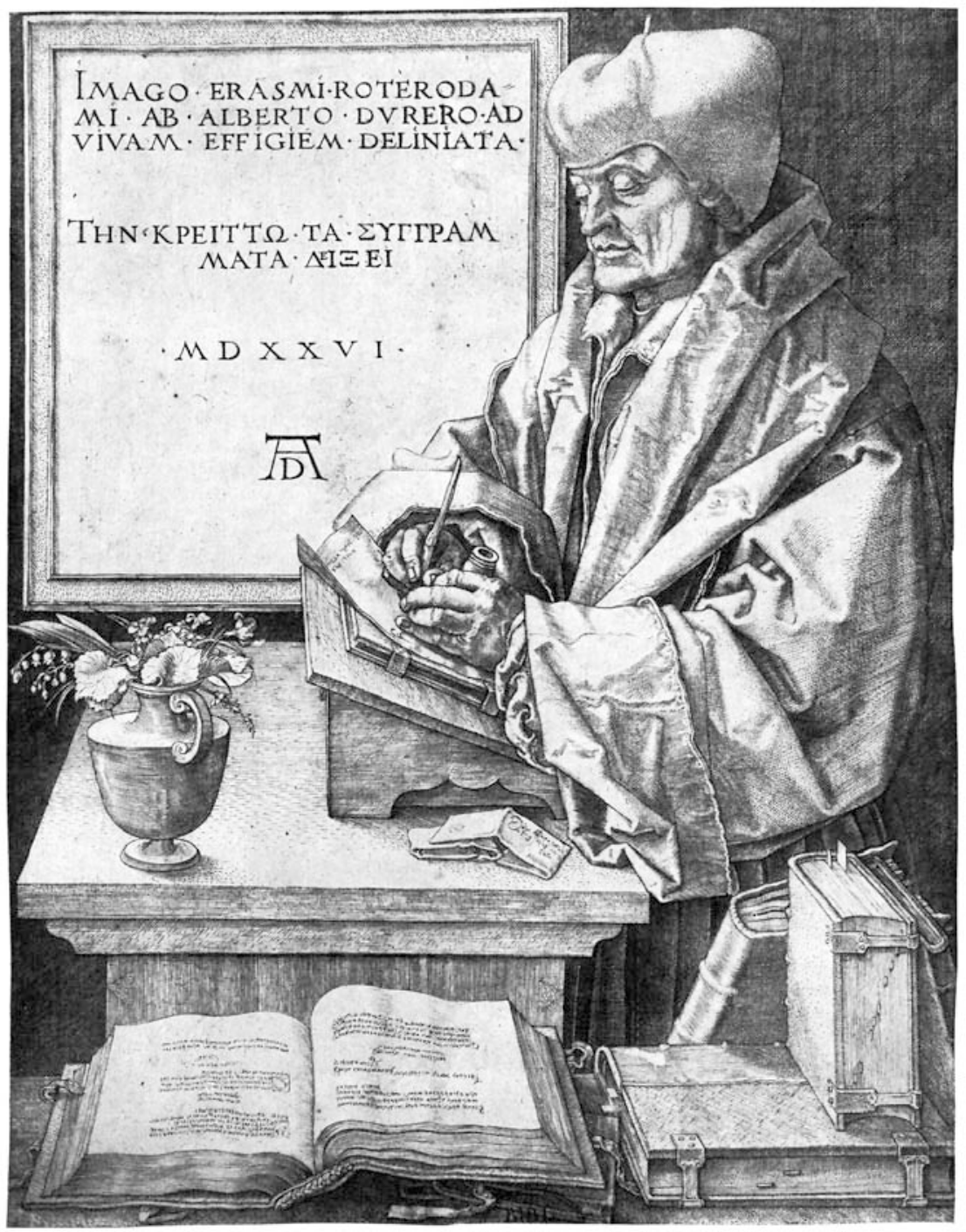

Abb. 1

Albrecht Dürer, Erasmus von Rotterdam, 1526

Kupferstich, 24,7 $\times 19,1 \mathrm{~cm}$

(Berlin, Staatliche Museen, Kupferstichkabinett) 


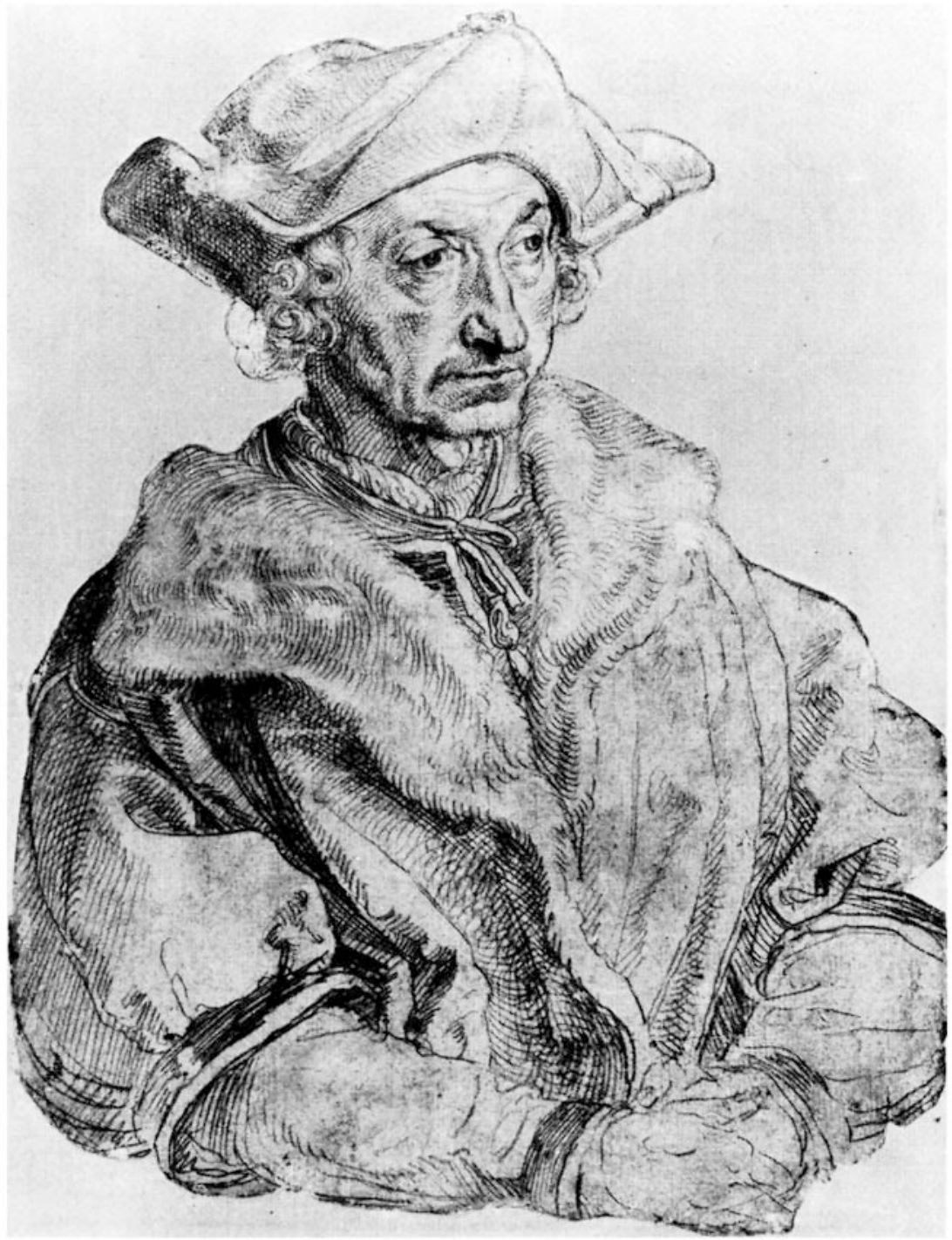

Abb. 2

Albrecht Dürer, Porträt des Sebastian Brant, 1520

Silberstiftzeichnung, 19,4 × 14,7 cm

(Berlin, Staatliche Museen, Kupferstichkabinett) 


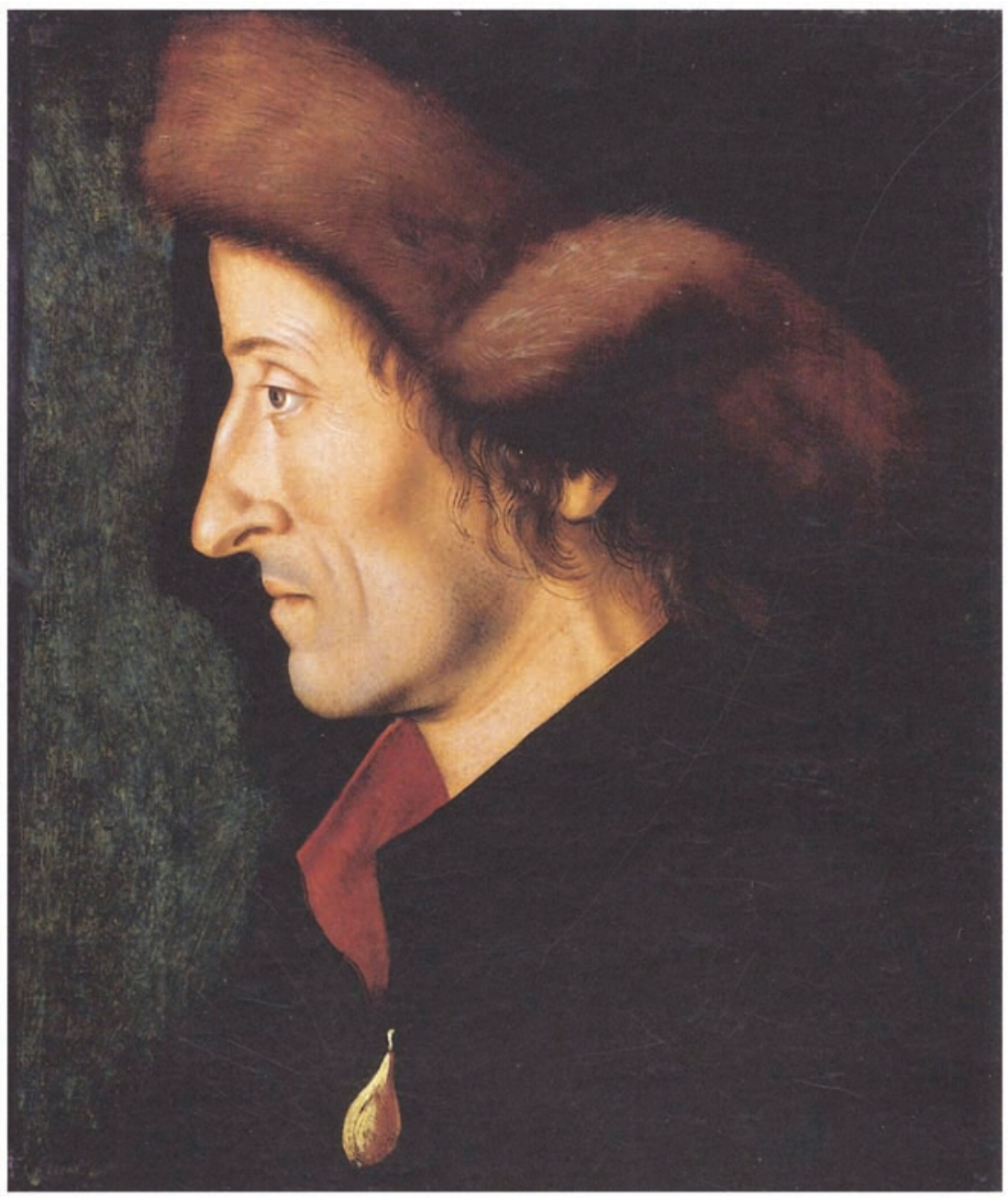

Abb. 3

Hans Burgkmair, Sebastian Brant, um 1508

Öl auf Leinwand, $37,5 \mathrm{~cm} \times 31,5 \mathrm{~cm}$

(Karlsruhe, Staatliche Kunsthalle) 


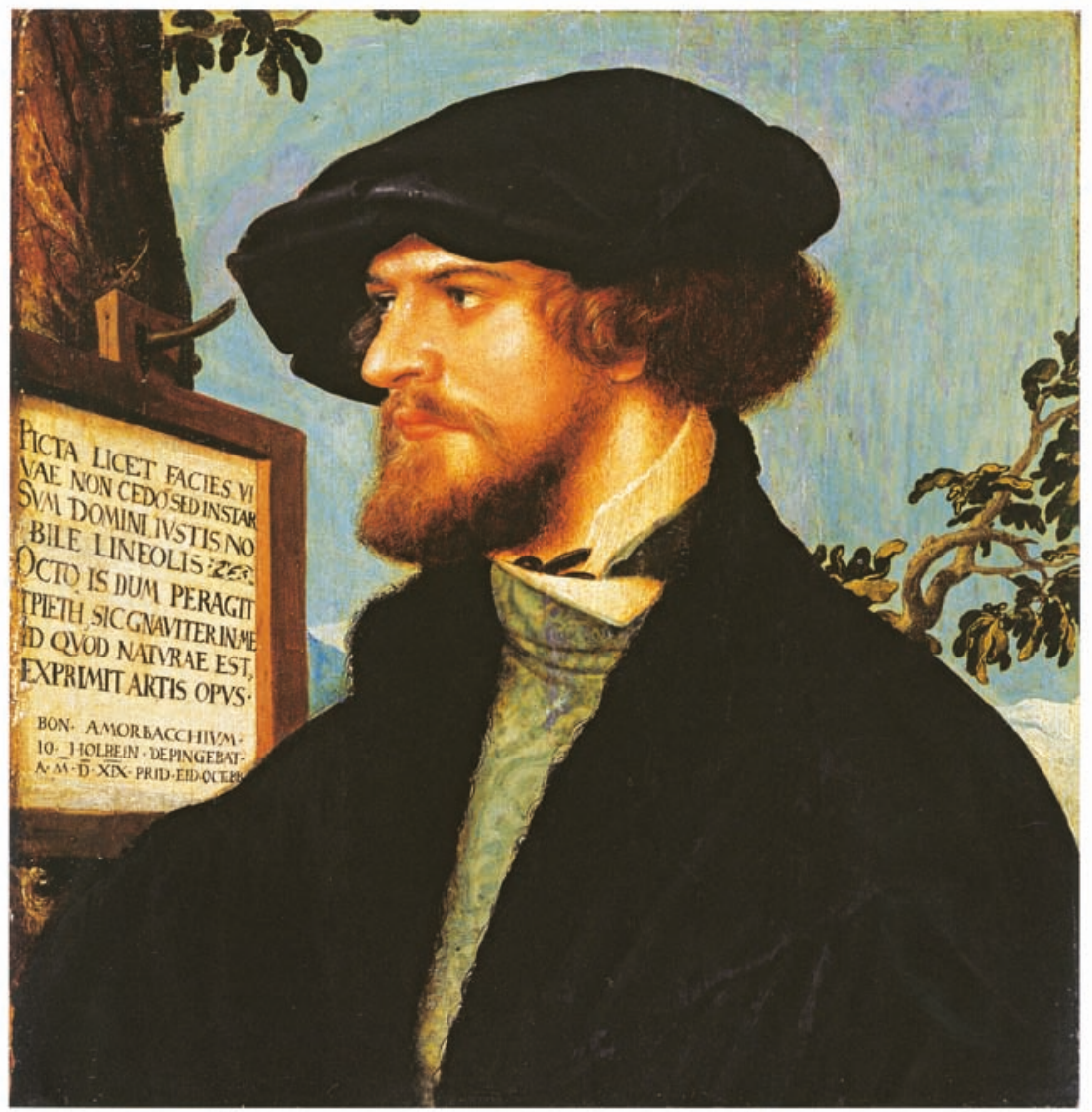

Abb. 4

Hans Holbein d. J., Bildnis des Bonifacius Amerbach, 1519 Öl auf Holz, 28,5 × 27,5 cm

(Basel, Kunstmuseum) 


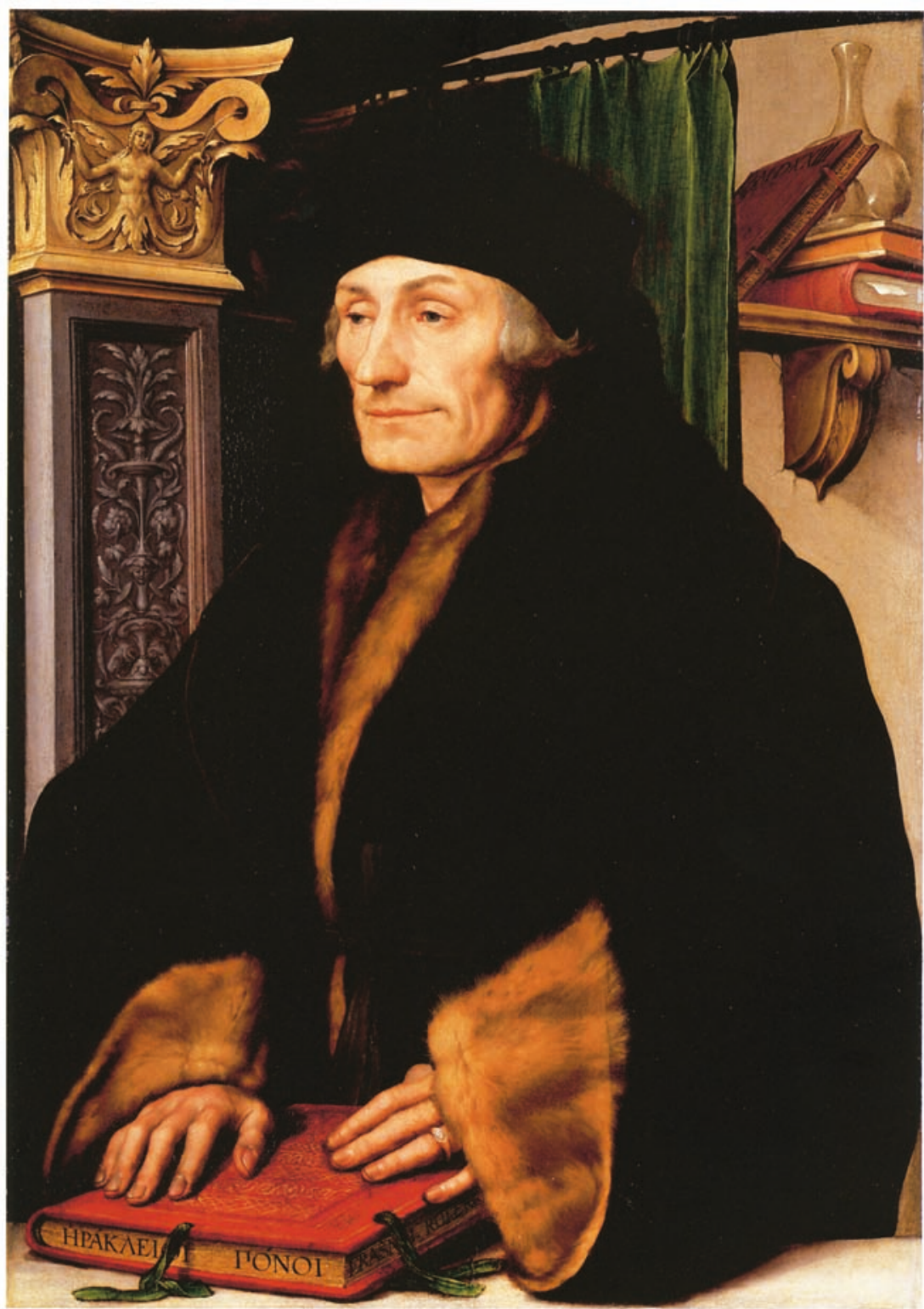

Abb. 5

Hans Holbein d. J., Erasmus von Rotterdam (Erasmus mit einem Renaissancepilaster), 1523, Öl auf Holz, $76 \times 51 \mathrm{~cm}$ (London, National Gallery, Leihgabe aus Privatbesitz) 


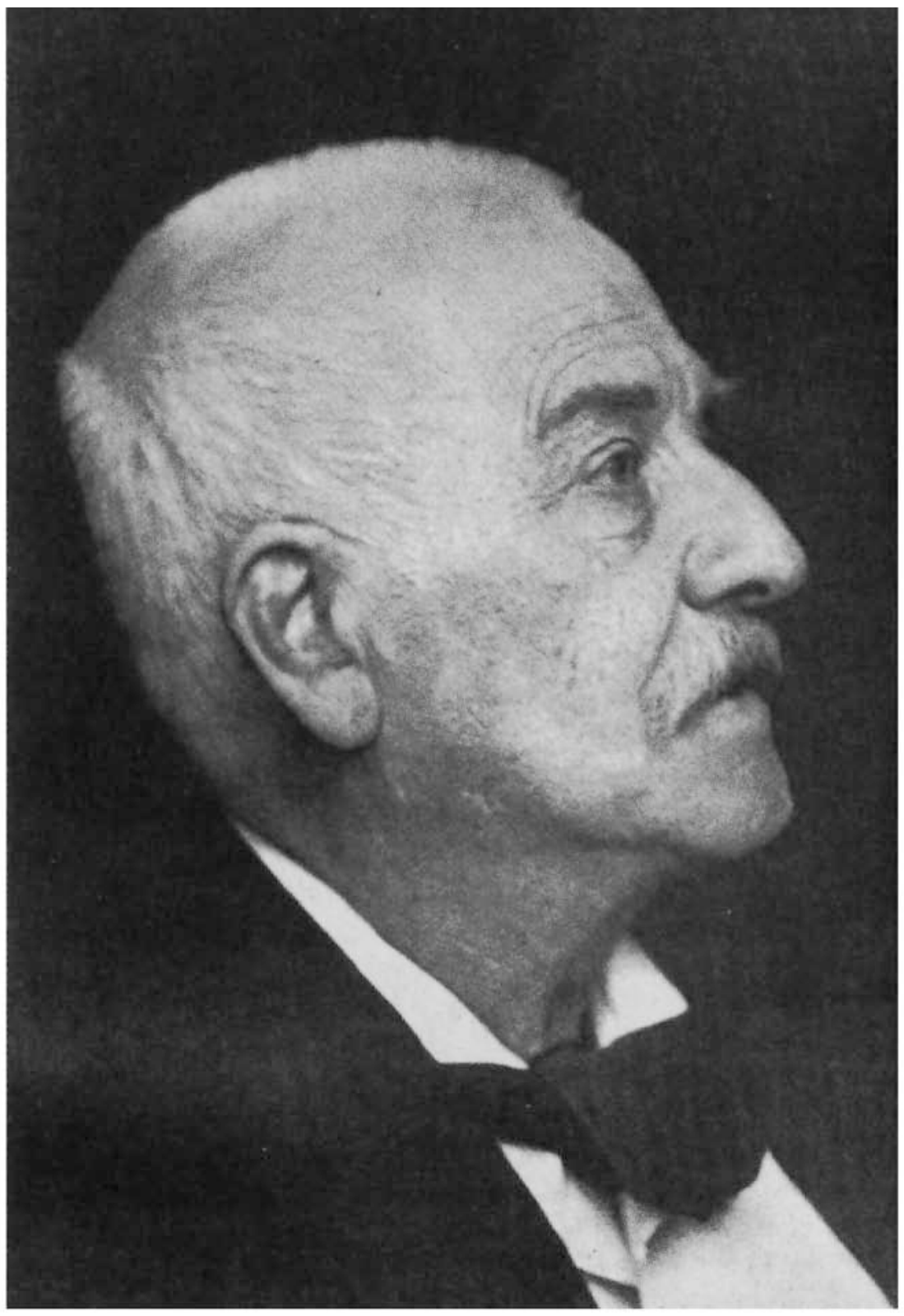

Abb. 6

Jacob Burckhardt 1892

(Jacob Burckhardt-Archiv, Universitätsbibliothek Basel) 